\title{
Agreement between Scheimpflug Camera and the Swept-source Optical Coherence Tomography Measurements in Keratometry and Higher-order Aberrations
}

\author{
Yujin Gim¹, Roo Min Jun², Kyung Eun Han $^{1}$ \\ ${ }^{1}$ Institute of Ophthalmology and Optometry, Department of Ophthalmology, Ewha Womans University Mokdong Hospital, Ewha \\ Womans University College of Medicine, Seoul, Korea \\ ${ }^{2}$ Institute of Ophthalmology and Optometry, Department of Ophthalmology, Ewha Womans University Seoul Hospital, Ewha Womans \\ University College of Medicine, Seoul, Korea
}

Purpose: To evaluate the compatibility of corneal curvature and astigmatism, and higher-order aberrations (HOAs) measured by the Scheimpflug camera Pentacam HR and the swept-source optical coherence tomography ANTERION.

Methods: This prospective study included normal subjects with no ophthalmic history. Steep keratometry (K), flat K, astigmatism and its axis of the anterior and posterior surfaces, total corneal power, and HOAs using the two instruments were compared. To compare the mean values of the measurements, a paired $t$-test was used. Bland-Altman analysis was applied to assess the agreement between the two devices.

Results: Fifty-three eyes of 53 subjects were evaluated. There were statistically significant differences for steep K, astigmatism, and vector J0, J45 in the anterior surface and total corneal power between the two devices $(p<0.05)$. There were also significant differences in the most of the keratometric values of the posterior corneal surface $(p<0.05)$ except J0 ( $p=$ 0.410). Both devices showed strong positive correlations in steep $K$, flat $K$, astigmatism $(r>0.81, p<0.001)$ with wide ranges of a 95\% limit of agreement. Vectoral components were significantly correlated $(r>0.78, p<0.001)$ with narrow $95 \%$ limit of agreement, except J45 of the posterior surface $(r=0.39, p=0.004)$. In the corneal HOAs, there were statistically significant differences in the vertical coma, horizontal trefoil, spherical aberration, and root mean square of each fifth- and sixth-order Zernike coefficient ( $p=0.043, p=0.041, p<0.001, p<0.001$, and $p<0.001$, respectively). Other HOAs showed moderate to strong positive correlations $(r>0.37, p<0.05)$. Most HOAs, except for the horizontal trefoil, showed clinically acceptable agreements. The total root mean square of HOAs was not significantly different between the two devices $(p=0.122)$.

Conclusions: Most of the keratometric values cannot be used interchangeably. However, the vectoral component of astigmatism showed clinically good agreement. Several HOAs have statistically significant differences; however, almost all HOAs showed acceptable agreements, except for the horizontal trefoil.

Key Words: Astigmatism, Corneal topography, Corneal wavefront aberration, Scheimpflug camera, Swept source optical coherence tomography

Received: May 14, $2021 \quad$ Final revision: June 25, $2021 \quad$ Accepted: June 30, 2021

Corresponding Author: Kyung Eun Han, MD, PhD. Department of Ophthalmology, Ewha Womans University Mokdong Hospital, 1071 Anyangcheon-ro, Yangcheon-gu, Seoul 07985, Korea. Tel: 82-2-2650-5154, Fax: 82-2654-4334, E mail: hanke@ewha.ac.kr

This paper was presented in online 125th Korean Ophthalmological Society for Cataract Refractive Surgery session. 
The measurement of the ocular anterior segment is essential in many clinical settings for the diagnosis of corneal diseases and perioperative evaluation for cataract surgery, corneal transplantation, and laser corneal refractive surgery. Several instruments using various principles, including the Scheimpflug camera, optical coherence tomography (OCT), Scheimpflug-Placido topography, and color light-emitting diode (LED) corneal topographer provide many measurements, such as anterior and posterior curvature, total corneal power (TCP), central corneal thickness, pupil diameter, white-to-white, anterior chamber depth, lens thickness, and corneal wavefront analysis [1-3].

Keratometry is one of the major measurements that can be acquired by those instruments. Recent studies have suggested that the use of total keratometry, including posterior corneal curvature, for intraocular lens (IOL) power calculation showed higher accuracy than conventional keratometry regarding anterior cornea curvature $[4,5]$. Other studies have reported the consideration of posterior corneal astigmatism when selecting a toric IOL can improve refractive outcomes after cataract surgery [6-9]. Thus, it is important to accurately measure and use corneal refractive power to achieve better results.

Corneal higher-order aberrations (HOAs) can be also measured with anterior segment instruments using wavefront analysis. HOAs importantly affect the quality of vision. Among the HOAs, coma, trefoil, and spherical aberration are known as visually significant aberrations; the vertical coma and spherical aberration are known as the two major HOAs that clinically significantly increase in keratoconus patients [10,11], and are related to visual acuity and discomfort after cataract surgery patients [12-14]. In addition, as the number of premium cataract surgeries using multifocal or toric IOL gains popularity in patients who had corneal refractive surgery, cut-off values of HOAs are suggested for those patients to prevent postoperative suboptimal visual outcome and patient's dissatisfaction [15]. Therefore, measuring corneal HOAs is essential for predicting and interpreting visual performance under various conditions.

The recently developed ANTERION (Heidelberg Engineering, Heidelberg, Germany) is a high-resolution sweptsource optical coherence tomography (SS-OCT) device that provides a variety of anterior segment metrics, including anterior, posterior, and total corneal curvatures, as well as HOAs with high-resolution, speed, providing an axial length, which calculates the IOL power as well. The high repeatability of anterior segment measurements including HOAs and axial length in ANTERION, has been reported [16-18], and the interchangeability in corneal curvature, white-to-white, pupil diameter, and anterior chamber depth between other devices such as IOL Master 700 (Carl Zeiss, Jena, Germany), Pentacam (Oculus, Wetzlar, Germany), and Cassini II (i-Optics, Den Haag, Netherlands) have been studied [19-22]. However, there is no comparative study on the HOAs of ANTERION with the Pentacam HR device. Accordingly, the purpose of this study is to confirm the agreement and compatibility of corneal curvature, astigmatism, and corneal HOAs measured by Pentacam HR and ANTERION.

\section{Materials and Methods}

\section{Subjects}

This prospective study was conducted following the Declaration of Helsinki and approved by the institutional review board of Ewha Womans University Medical Center (2021-01-005). The study was explained to all patients, and informed consent was obtained from all enrolled subjects. This study was conducted between February 15, 2021, and February 28, 2021, in adults aged 20 to 60 years of age without an ophthalmic history. The subjects who have ocular conditions such as keratoconjunctival diseases including dry eye syndrome and conjunctivochalasis, who had a history of laser corneal refractive surgery or ocular trauma, and who used contact lenses within one week were excluded. Subjects who showed an error on the measurements because of excessive blinking or who complained of ocular discomfort during the examination were also excluded.

\section{Devices}

The Pentacam HR has a Scheimpflug camera that rotates 360 degrees to obtain an elevation map of the anterior and posterior surfaces of the cornea. It analyzes the shape of the cornea and calculates the HOAs of the anterior and posterior surfaces, and total of the cornea. The wavefront aberration data are ray-traced calculated $[23,24]$. The ANTERION is a newly developed SS-OCT device that uses a 
A

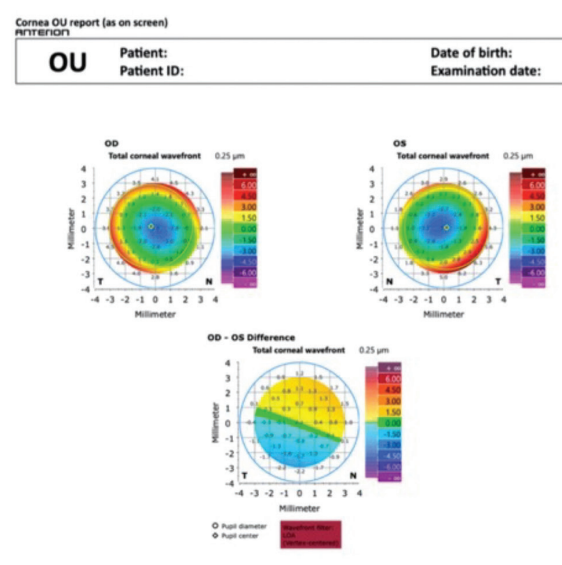

B

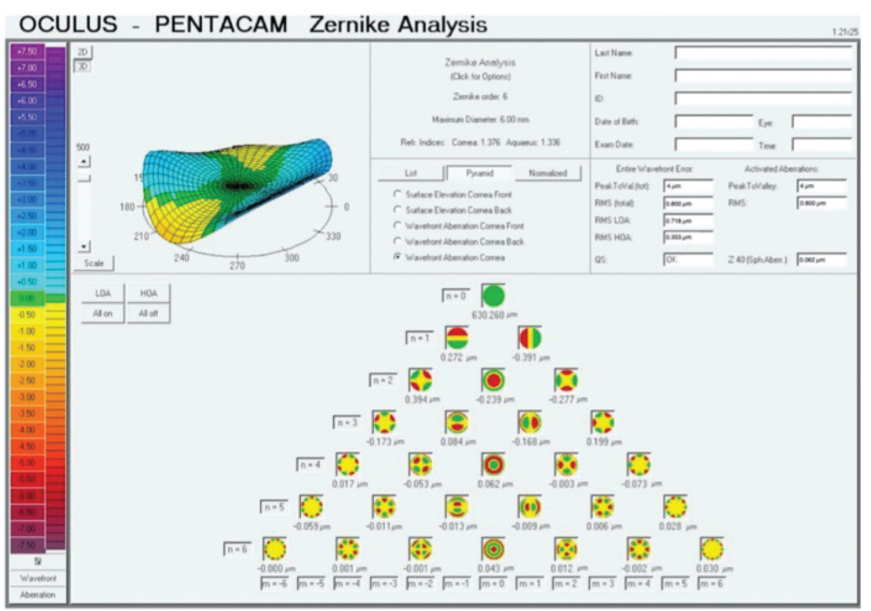

Fig. 1. Representative outcome images of wavefront analysis in (A) ANTERION and (B) Pentacam HR.

longer wavelength $(1,300 \mathrm{~nm})$ light source to offer high-resolution anterior segment biometry. All measurements are assisted by an eye-tracking technology, centered on the corneal vertex. A total of 65 radial B-scan images and 256 A-scans per B-scan are acquired quickly. Using the Cornea App mode, corneal topography and tomography, corneal wavefront analysis and pachymetry data can be measured within a few seconds. Anterior and total corneal wavefront error was calculated using ray tracing method [25].

\section{Measurement process and measured parameters}

One trained examiner performed the ocular examination, including visual acuity, slit lamp microscopy, autorefractor/ keratometer (ARK-510A; NIDEK, Gamagori, Japan), and two anterior segment imaging devices in the order of ANTERION and Pentacam HR in mesopic condition. Each test was performed after at least a 5-minute interval, and in all tests, the right eye was measured first. Both devices provide image quality assessment, so only cases that pass the quality evaluation were included in the study; quality factor provided by Pentacam HR is 'OK' and acquisition quality information provided by ANTERION, including motion, fixation, tear film and lid, camera image segmentation, refraction correction and required data point shows 'pass'. A slit lamp examination was performed using a fluorescein strip (Fluorescein Strip; Haag-Streit USA, Mason, OH, USA) to evaluate the tear break up time and corneal pathology.
For corneal keratometry, steep keratometry (Ks), flat keratometry (Kf), and astigmatism (Kastig) in the anterior, posterior curvature, and TCP were compared between the two devices. The keratometry for the steep and flat meridian of the anterior and posterior cornea was measured within the central 3-mm zone in the two devices. The TCP was also measured by ray tracing with a diameter of $3 \mathrm{~mm}$ in two instruments. The power vector analysis of astigmatism was performed using the following formulae based on the method described by Thibos et al. [26] and Thibos and Horner [27]. (1) $\mathrm{J} 0=[-\mathrm{C} / 2 \times \operatorname{Cos} 2 \theta](2) \mathrm{J} 45=[-\mathrm{C} / 2 \times \operatorname{Sin} 2 \theta]$, where $\mathrm{C}$ is negative cylindrical power ( $\mathrm{Kf}-\mathrm{Ks})$ and $\theta$ is the cylindrical axis (flat meridian). J0 is the power of Jackson cross-cylinder power vector at 90- and 180-degrees axes, thus positive values of $\mathrm{J} 0$ indicate with-the-rule astigmatism. J45 is the power of the Jackson cross-cylinder power vector at 45- and 135-degrees axes. These two vectoral components, J0 and J45, were also compared for anterior, posterior, and total corneal astigmatism in a $3.0-\mathrm{mm}$ zone.

In corneal HOAs, each Zernike coefficient of oblique trefoil, vertical coma, horizontal coma, horizontal trefoil, and spherical aberration were compared at the diameters of a 6-mm zone. The root mean square (RMS) of each third to sixth order Zernike coefficients and the RMS of total HOAs were also analyzed. In the Zernike polynomial coefficient, the result sheet of the ANTERION device provides up to one decimal place and that of Pentacam HR up to three decimal places, so the results of Pentacam HR are rounded up to one decimal place for comparison (Fig. 1A, 1B). 


\section{Statistical analysis}

Statistical analysis was performed using IBM SPSS Statistics ver. 20.0 (IBM Corp., Armonk, NY, USA). To compare the mean values of measurements were accessed with

Table 1. Demographics of subject and autorefractor/keratometer measurements

\begin{tabular}{lc}
\hline Characteristics & Value \\
\hline Age (yr) & $36 \pm 9(19-59)$ \\
Sex (male : female) & $22: 31$ \\
Autorefractor/keratometry (D) & \\
Spherical equivalent & $-3.31 \pm 3.26(-12.75$ to +2.75$)$ \\
Steep keratometry & $43.84 \pm 1.53(40.75$ to 47.50$)$ \\
Flat keratometry & $42.76 \pm 1.42(39.50$ to 45.75$)$ \\
Corneal astigmatism & $1.08 \pm 0.50(0$ to 2.25$)$ \\
\hline
\end{tabular}

Values are presented as mean \pm standard deviation (range) or number.

$\mathrm{D}=$ diopter. a paired $t$-test and to evaluate the correlation of parameters, Pearson's correlation tests were performed. Bland-Altman plots were used to analyze the agreements between the two devices, and the $95 \%$ limits of agreement (LoA) were obtained. The LoA was calculated as the mean difference \pm 1.96 standard deviation. A $p$-value of less than 0.05 was considered statistically significant.

\section{Results}

\section{Demographics}

A total of 53 eyes of 53 patients ( 22 males and 31 females) without ocular pathology were included. The age range was from 19 to 59 years with a mean of $36 \pm 9$ years. Of the 53 eyes, five eyes were analyzed for the left eye, and 48 eyes were analyzed for the right eye due to the incomplete measurement of the HOAs by the ANTERION device. The

Table 2. Comparison of ANTERION and Pentacam HR measurements in keratometry and astigmatism

\begin{tabular}{|c|c|c|c|c|}
\hline & ANTERION & Pentacam HR & Mean difference & $p$-value \\
\hline \multicolumn{5}{|c|}{ Anterior corneal curvature (D) } \\
\hline Ks & $43.67 \pm 1.49$ & $43.79 \pm 1.53$ & $-0.12 \pm 0.19$ & $<0.001$ \\
\hline $\mathrm{Kf}$ & $42.63 \pm 1.37$ & $42.64 \pm 1.41$ & $-0.01 \pm 0.20$ & 0.687 \\
\hline Kastig & $1.04 \pm 0.52$ & $1.14 \pm 0.53$ & $-0.10 \pm 0.17$ & $<0.001$ \\
\hline J0 & $0.45 \pm 0.31$ & $0.52 \pm 0.31$ & $-0.07 \pm 0.10$ & $<0.001$ \\
\hline $\mathrm{J} 45$ & $-0.10 \pm 0.16$ & $0.01 \pm 0.19$ & $-0.09 \pm 0.10$ & $<0.001$ \\
\hline \multicolumn{5}{|c|}{ Posterior corneal curvature (D) } \\
\hline Ks & $-6.35 \pm 0.25$ & $-6.48 \pm 0.26$ & $0.13 \pm 0.07$ & $<0.001$ \\
\hline $\mathrm{Kf}$ & $-5.98 \pm 0.23$ & $-6.10 \pm 0.24$ & $0.12 \pm 0.07$ & $<0.001$ \\
\hline Kastig & $-0.38 \pm 0.11$ & $-0.36 \pm 0.13$ & $-0.02 \pm 0.08$ & 0.027 \\
\hline $\mathrm{J} 0$ & $-0.18 \pm 0.06$ & $-0.18 \pm 0.06$ & $0.00 \pm 0.04$ & 0.410 \\
\hline $\mathrm{J} 45$ & $0.02 \pm 0.04$ & $-0.01 \pm 0.05$ & $0.03 \pm 0.05$ & $<0.001$ \\
\hline \multicolumn{5}{|l|}{ TCP (D) } \\
\hline TCPs & $43.00 \pm 1.51$ & $43.12 \pm 1.54$ & $-0.11 \pm 0.25$ & 0.002 \\
\hline TCPf & $42.13 \pm 1.38$ & $42.07 \pm 1.42$ & $0.05 \pm 0.24$ & 0.058 \\
\hline TCPastig & $0.87 \pm 0.49$ & $1.03 \pm 0.52$ & $-0.17 \pm 0.20$ & $<0.001$ \\
\hline $\mathrm{J} 0$ & $0.34 \pm 0.32$ & $0.44 \pm 0.33$ & $-0.10 \pm 0.11$ & $<0.001$ \\
\hline $\mathrm{J} 45$ & $-0.10 \pm 0.16$ & $0.00 \pm 0.20$ & $-0.09 \pm 0.09$ & $<0.001$ \\
\hline
\end{tabular}

Values are presented as mean \pm standard deviation.

$\mathrm{D}=$ diopter; $\mathrm{Ks}=$ curvature power of the steep meridian of cornea; $\mathrm{Kf}=$ curvature power of the flat meridian of cornea; Kastig = corneal astigmatism; TCP = total corneal power; TCPs = TCP of the steep meridian of cornea; TCPf $=$ TCP of the flat meridian of cornea; TCPastig $=$ astigmatism of TCP; J0 = corneal astigmatism vector at the 90- and 180-degree axes; J45 = corneal astigmatism vector at the 45- and 135-degree axes.

*Paired t-test used; $p<0.05$ were considered statistically significant. 
mean spherical equivalent measured by the autorefractor/ keratometer was $-3.31 \pm 3.26$ diopters (D) (Table 1).

\section{Keratometry and TCP}

Table 2 shows the mean values and differences in keratometric measurements between the two devices. Of the anterior corneal curvature, there was a statistically significant difference in Ks and Kastig ( $p<0.001)$, but not in $\mathrm{Kf}$ ( $p=$ 0.687). All posterior corneal measurements including Ks, $\mathrm{Kf}$, and Kastig showed statistically significant differences between the two devices $(p<0.001)$. In TCP, like the results of anterior corneal curvature, statistically significant differences were observed in TCP of the steep meridian of cornea $(p=0.002)$ and astigmatism of TCP $(p<0.001)$, but not in TCP of the flat meridian of cornea $(p=0.058)$. Vectoral component analysis of astigmatism revealed statistically significant differences in J0 and J45 of anterior, posterior, and total astigmatism $(p<0.001)$ except $\mathrm{J} 0$ of posterior astigmatism $(p=0.410)$. There were strong positive correlations in $\mathrm{Ks}, \mathrm{Kf}$, Kastig between the two devices ( $\mathrm{r}>0.81, p$ $<0.001$ ) (Table 3). However, for the J45 vector of posterior astigmatism, there was a weak positive correlation between the two devices ( $\mathrm{r}=0.39, p=0.004)$. Other vectoral components were significantly correlated showing strong positive correlation ( $\mathrm{r}>0.78, p<0.001$ ) (Table 3). Bland-Altman analyses for $\mathrm{Ks}, \mathrm{Kf}$, and Kastig in anterior and posterior corneal curvatures and TCP between ANTERION and Pentacam HR showed clinically poor agreement. However, J0 and J45 measurements showed clinically good agreement with narrow LoA range in anterior and posterior, total astigmatism (Table 3 and Fig. 2A-2O).

\section{Higher-order aberrations}

There were statistically significant differences in the vertical coma, horizontal trefoil, spherical aberration, and RMS of the fifth and sixth order $(p=0.043, p=0.041, p<$

Table 3. Correlation and agreement of keratometry between ANTERION and Pentacam HR

\begin{tabular}{lllll}
\hline & Correlation coefficient & $p$-value & 95\% LoA \\
\hline Anterior corneal curvature & & & & \\
Ks (D) & 0.99 & $<0.001$ & -0.17 to -0.07 & -0.50 to 0.26 \\
Kf (D) & 0.99 & $<0.001$ & -0.07 to 0.04 & -0.41 to 0.39 \\
Kastig (D) & 0.95 & $<0.001$ & -0.15 to -0.05 & -0.44 to 0.24 \\
J0 & 0.95 & $<0.001$ & -0.10 to -0.04 & -0.27 to 0.13 \\
J45 & 0.85 & $<0.001$ & -0.13 to -0.07 & -0.29 to 0.10 \\
Posterior corneal curvature & & & 0.00 to 0.25 \\
Ks (D) & 0.97 & $<0.001$ & 0.11 to 0.14 & -0.01 to 0.25 \\
Kf (D) & 0.96 & $<0.001$ & 0.10 to 0.14 & -0.18 to 0.13 \\
Kastig (D) & 0.81 & $<0.001$ & -0.05 to 0.00 & -0.07 to 0.08 \\
J0 & 0.78 & -0.01 to 0.02 & -0.07 to 0.12 \\
J45 & 0.39 & 0.001 & 0.01 to 0.04 & -0.61 to 0.39 \\
TCP & & 0.004 & & -0.42 to 0.54 \\
TCPs (D) & 0.99 & $<0.001$ & -0.19 to -0.05 & -0.56 to 0.23 \\
TCPf (D) & 0.99 & $<0.001$ & 0.00 to 0.13 & -0.32 to 0.11 \\
TCPastig (D) & 0.92 & $<0.001$ & -0.22 to -0.11 & -0.28 to 0.09 \\
J0 & 0.94 & $<0.001$ & -0.12 to -0.07 & \\
J45 & 0.89 & $<0.001$ & & \\
\hline
\end{tabular}

$\mathrm{D}=$ diopter; $\mathrm{CI}=$ confidence interval; $\mathrm{LoA}=$ limits of agreement; $\mathrm{Ks}=$ curvature power of the steep meridian of cornea; $\mathrm{Kf}=$ curvature power of the flat meridian of cornea; Kastig = corneal astigmatism; TCP $=$ total corneal power; TCPs $=$ TCP of the steep meridian of cornea; TCPf $=$ TCP of the flat meridian of cornea; TCPastig = astigmatism of TCP; J0 = corneal astigmatism vector at the 90 - and 180-degree axes; J45 = corneal astigmatism vector at the 45- and 135-degree axes.

"Pearson correlation test used; $p<0.05$ were considered statistically significant. 

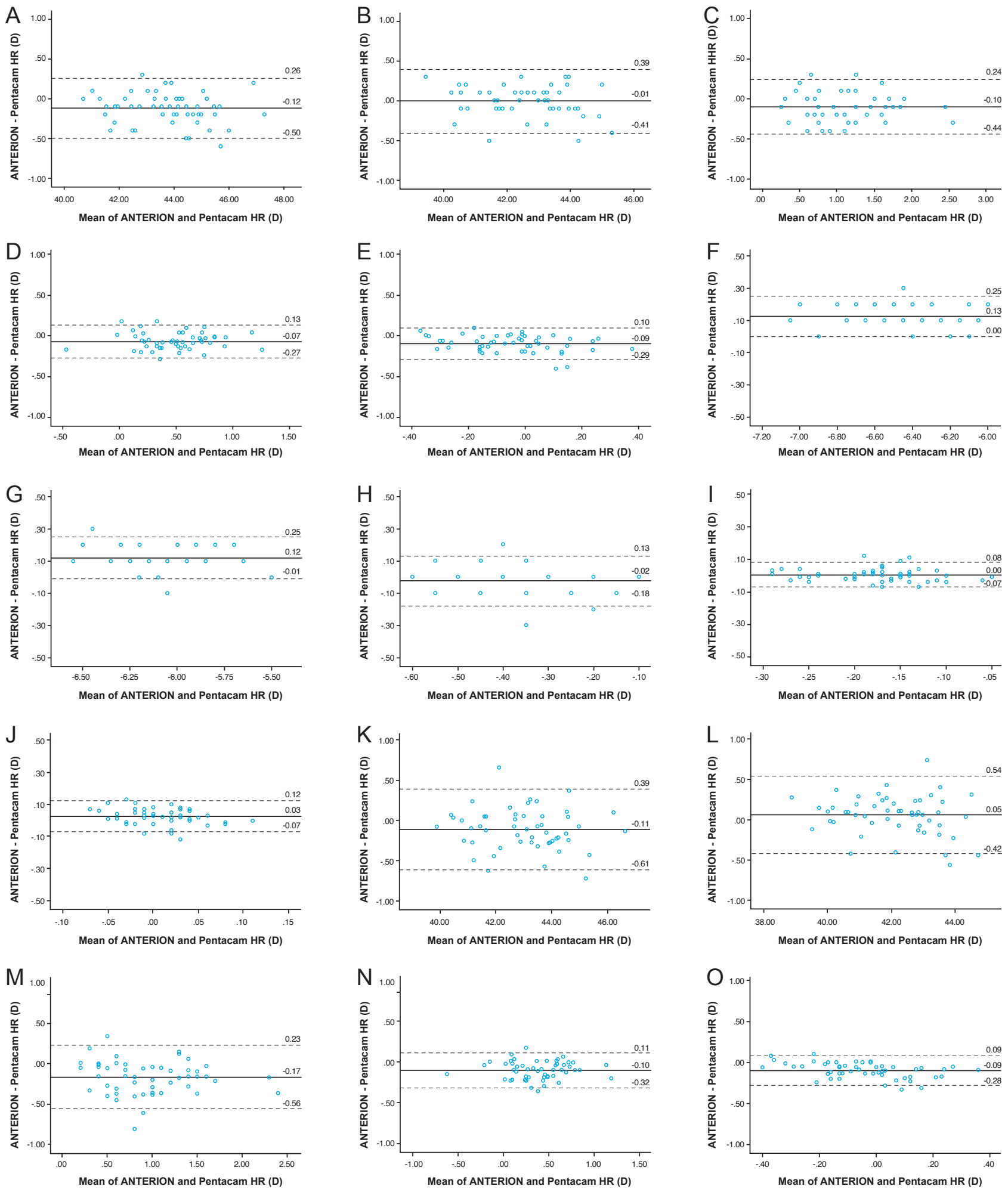

Fig. 2. Agreement of keratometry and astigmatism between ANTERION and Pentacam HR. Solid line showed the mean difference, and the other line showed the $95 \%$ limits of agreement. (A) Anterior steep keratometry. (B) Anterior flat keratometry. (C) Anterior corneal astigmatism. (D) Anterior vector J0. (E) Anterior vector J45. (F) Posterior steep keratometry. (G) Posterior flat keratometry. (H) Posterior corneal astigmatism. (I) Posterior vector J0. (J) Posterior vector J45. (K) Total corneal power of steep meridian. (L) Total corneal power of flat meridian. (M) Total corneal power astigmatism. (N) Total vector J0. (O) Total vector J45. D = diopter. 
0.001 , and $p<0.001$, respectively) (Table 4). There was a significant positive correlation in almost all HOAs, but not in the horizontal trefoil and RMS of the fifth and sixth order (Table 5). The total RMS of HOAs was measured at $0.43 \pm 0.12 \mu \mathrm{m}$ for ANTERION and $0.40 \pm 0.13 \mu \mathrm{m}$ for Pentacam HR, and there was no statistically significant difference $(p=0.122)$. Fig. 3A-3J shows the Bland-Altman plots for HOAs. The mean differences (95\% LoA range) were $-0.01 \pm 0.17 \mu \mathrm{m}(-0.35$ to 0.33$)$ for oblique trefoil, $-0.04 \pm 0.13 \mu \mathrm{m}$ ( -0.31 to 0.23$)$ for the vertical coma, $0.00 \pm$ $0.08 \mu \mathrm{m}(-0.15$ to 0.17$)$ for the horizontal coma, $-0.05 \pm 0.18$ $\mu \mathrm{m}(-0.43$ to 0.32$)$ for the horizontal trefoil, $-0.05 \pm 0.18 \mu \mathrm{m}$ $(-0.09$ to 0.24$)$ for the spherical aberration, and $0.02 \pm 0.14$ $\mu \mathrm{m}(-0.24$ to 0.30$)$ for the total RMS of HOAs. There was no systemic or proportional bias in the Bland-Altman analysis in keratometry and HOAs.

Table 4. Comparison of higher-order aberrations between ANTERION and Pentacam HR

\begin{tabular}{lcccc}
\hline & ANTERION & Pentacam HR & Mean difference & $p$-value \\
\hline Third order & & & & \\
$\quad$ Oblique trefoil & $-0.09 \pm 0.18$ & $-0.08 \pm 0.13$ & $-0.01 \pm 0.17$ & 0.697 \\
$\quad$ Vertical coma & $-0.06 \pm 0.20$ & $-0.03 \pm 0.20$ & $-0.04 \pm 0.13$ & 0.043 \\
Horizontal coma & $-0.08 \pm 0.12$ & $-0.09 \pm 0.13$ & $-0.00 \pm 0.08$ & 0.617 \\
Horizontal trefoil & $-0.03 \pm 0.13$ & $-0.02 \pm 0.16$ & & 0.041 \\
Fourth order & & & $0.07 \pm 0.08$ & $<0.001$ \\
$\quad$ Spherical aberration & $0.23 \pm 0.13$ & $0.15 \pm 0.10$ & $0.02 \pm 0.15$ & 0.366 \\
RMS of the third order & $0.33 \pm 0.11$ & $0.30 \pm 0.14$ & $0.02 \pm 0.09$ & 0.063 \\
RMS of the fourth order & $0.26 \pm 0.11$ & $0.23 \pm 0.10$ & $-0.05 \pm 0.07$ & $<0.001$ \\
RMS of the fifth order & $0.04 \pm 0.05$ & $0.10 \pm 0.06$ & $-0.07 \pm 0.04$ & $<0.001$ \\
RMS of the sixth order & $0.00 \pm 0.02$ & $0.08 \pm 0.05$ & $0.02 \pm 0.14$ & 0.122 \\
Total RMS HOAs & $0.43 \pm 0.12$ & $0.40 \pm 0.13$ & & \\
\hline
\end{tabular}

Values are presented as mean \pm standard deviation.

$\mathrm{RMS}=$ root mean square; HOAs $=$ higher-order aberrations.

${ }^{*}$ Paired $t$-test; $p<0.05$ were considered statistically significant.

Table 5. Agreement of higher-order aberrations between ANTERION and Pentacam HR

\begin{tabular}{|c|c|c|c|c|}
\hline & $\begin{array}{l}\text { Correlation } \\
\text { coefficient, }(\mathrm{r})\end{array}$ & $p$-value ${ }^{*}$ & $95 \% \mathrm{CI}$ & $95 \%$ LoA \\
\hline \multicolumn{5}{|l|}{ Third order } \\
\hline Oblique trefoil & 0.41 & 0.002 & -0.06 to 0.04 & -0.35 to 0.33 \\
\hline Vertical Coma & 0.77 & $<0.001$ & -0.08 to 0.00 & -0.31 to 0.23 \\
\hline Horizontal Coma & 0.79 & $<0.001$ & -0.02 to 0.02 & -0.15 to 0.17 \\
\hline Horizontal trefoil & 0.18 & 0.192 & -0.11 to 0.00 & -0.43 to 0.32 \\
\hline \multicolumn{5}{|l|}{ Fourth order } \\
\hline Spherical aberration & 0.76 & $<0.001$ & 0.05 to 0.09 & -0.09 to 0.24 \\
\hline RMS of the third order & 0.37 & 0.006 & -0.03 to 0.06 & -0.29 to 0.32 \\
\hline RMS of the fourth order & 0.60 & $<0.001$ & 0.00 to 0.05 & -0.16 to 0.21 \\
\hline RMS of the fifth order & 0.14 & 0.181 & -0.07 to -0.03 & -0.21 to 0.10 \\
\hline RMS of the sixth order & 0.10 & 0.623 & -0.08 to -0.06 & -0.17 to 0.03 \\
\hline Total RMS & 0.42 & $<0.001$ & -0.01 to 0.07 & -0.24 to 0.30 \\
\hline
\end{tabular}

$\mathrm{CI}=$ confidence interval; $\mathrm{LoA}=$ limits of agreement; $\mathrm{RMS}=$ root mean square; HOAs $=$ higher-order aberrations *Pearson correlation; $p<0.05$ were considered statistically significant. 
A

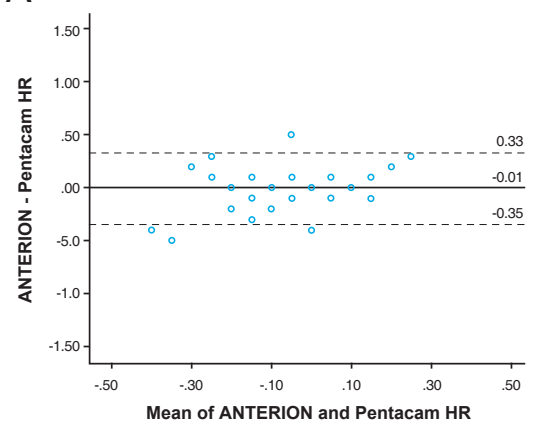

D
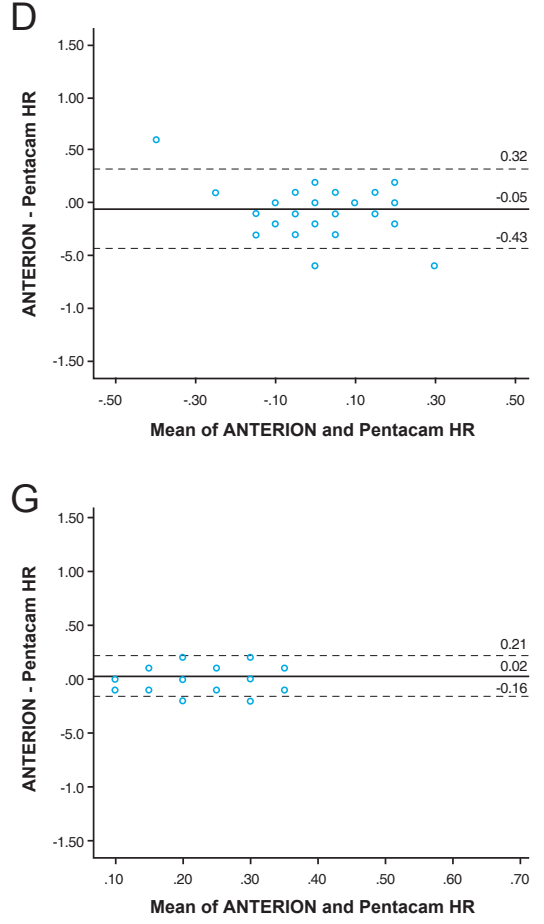

$J$

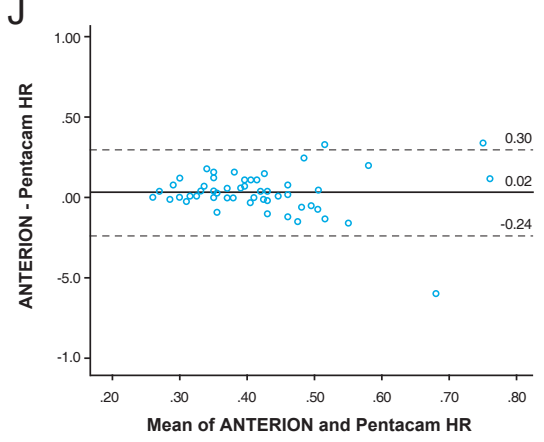

B

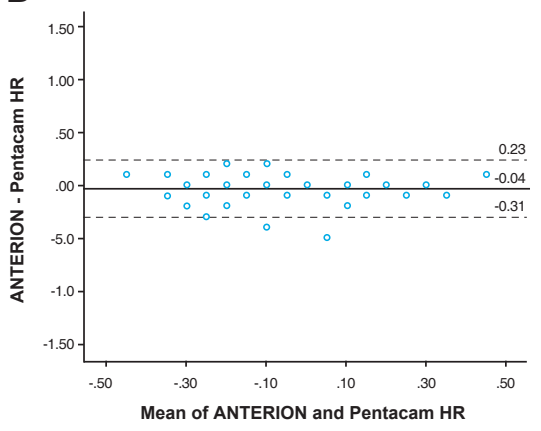

$E$

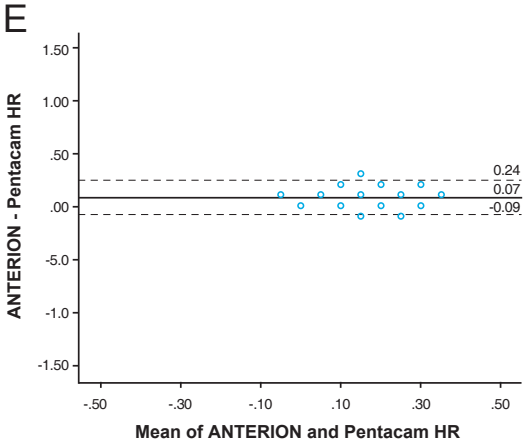

$\mathrm{H}$

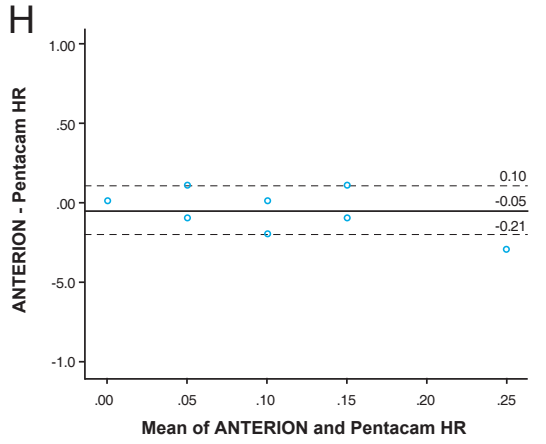

C

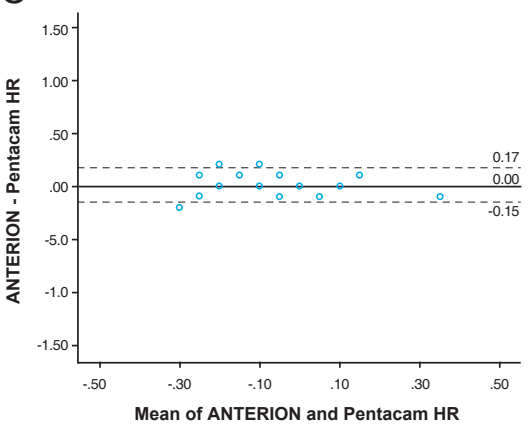

$\mathrm{F}$

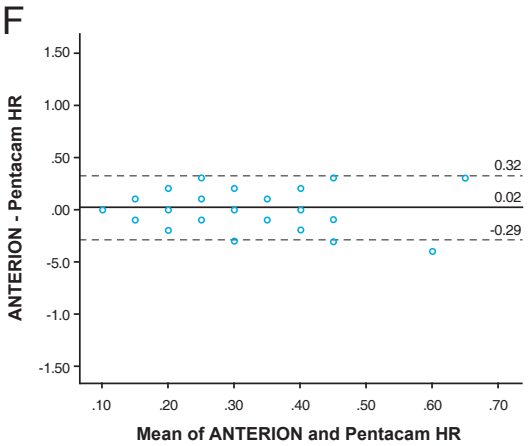

1

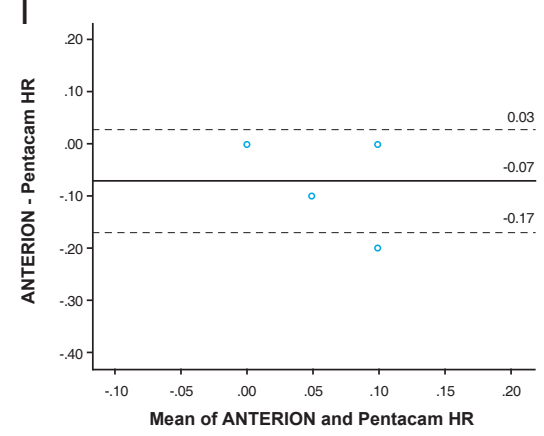

Fig. 3. Agreement of higher-order aberrations between ANTERION and Pentacam HR. Solid line showed the mean difference, and the other line showed the 95\% limits of agreement. (A) Oblique trefoil. (B) Vertical coma. (C) Horizontal coma. (D) Horizontal trefoil. (E) Spherical aberration. (F) Root mean square (RMS) of the third order. (G) RMS of the fourth order. (H) RMS of the fifth order. (I) RMS of the sixth order. (J) Total RMS of higher-order aberrations. 


\section{Discussion}

In this study, we assessed the agreement of keratometric measurements including power vector analysis of astigmatism in anterior and posterior cornea curvature, TCP, and corneal HOAs obtained by the Scheimpflug camera Pentacam HR and SS-OCT based-device ANTERION. There were statistically significant differences in most values except the anterior Kf, TCP of the flat meridian of cornea, and posterior J0. Most of the keratometric measurements showed poor agreement, which is difficult to use interchangeably. However, J0 and J45 values can be used interchangeable, showing mean differences $<0.1 \mathrm{D}$ and narrow ranges of $95 \%$ LoA. Among the corneal HOAs, vertical coma, horizontal trefoil, spherical aberration, and each RMS of the fifth and sixth order showed statistically significant differences and the horizontal trefoil and RMS of the fifth and sixth order showed no significant correlation. However, almost all HOAs showed clinically acceptable agreements with a narrow 95\% LoA range.

There have been many efforts to reduce residual refractive error after ocular surgery such as cataract or refractive surgery, and this is closely related to the accurate assessment of the ocular biometrics. Recent studies have shown the importance of the considering posterior corneal astigmatism and total keratometry for cataract surgery, as it has been demonstrated to minimize postoperative refractive errors [4-9]. The Scheimpflug device has been used to measure both anterior and posterior corneal curvature, and the Pentacam HR showed high accuracy for total corneal astigmatism [28]. Park et al. [29] reported that when selecting the appropriate toric IOL cylinder power, vector summation using the anterior and posterior corneal curvature measured by Pentacam HR showed decreased prediction error for astigmatism, and was superior to IOL Master keratometry and simulated keratometry from Pentacam HR. As such, since the clinical usefulness of Pentacam HR for keratometry and utilization has already been proven, it is important to evaluate compatibility with Pentacam HR for other newly developed devices.

In other studies of inter-device comparison for keratometry, Ozyol et al. [30] assessed the agreement between Pentacam HR and IOL Master 700 in 62 eyes of 62 patients. When comparing the mean keratometry $(\mathrm{Km})$ of the IOL Master 700 of the $2.5-\mathrm{mm}$ zone with that of the Pentacam $\mathrm{HR}$ at the 2- and 3-mm zone, there was a statistically sig- nificant difference between the two devices $(p<0.001)$, and the $95 \%$ LoA ranged from -0.45 to $0.17 \mathrm{D}$ in the $2-\mathrm{mm}$ zone and -0.38 to $-0.02 \mathrm{D}$ in the 3 -mm zone. These differences are sufficient to cause refractive errors when calculating the IOL power. However, there were agreements on the $\mathrm{J} 0$ and $\mathrm{J} 45$ vectoral components of astigmatism of simulated keratometry, showing that the mean difference was $0.07 \pm 0.09 \mathrm{D}$ with a $95 \% \mathrm{LoA}$ of -0.10 to 0.24 for $\mathrm{J} 0$ and $-0.016 \pm 0.15 \mathrm{D}$ with a $95 \%$ LoA of -0.31 to 0.27 for J45. Cui et al. [31] compared keratometric measurements using the Pentacam HR and Cassini in 117 eyes of 117 patients before cataract surgery. The statistically significant differences were observed in $\mathrm{Km}$, astigmatism, and $\mathrm{J} 0$ for the anterior cornea and in $\mathrm{Kf}, \mathrm{Km}$, astigmatism, and $\mathrm{J} 0$ for the posterior cornea. In addition, the ranges of $95 \%$ LoA were large for most variables not to be used interchangeably, extending beyond $0.5 \mathrm{D}$ in most of the variables. Tana-Rivero et al. [22] compared the ocular biometry of 49 eyes using ANTERION, IOL Master 700, and Pentacam AXL (Oculus) and reported no statistically significant difference for anterior Ks and Kf. For the agreement between ANTERION and Pentacam AXL, the 95\% LoA range was wide (from -0.3643 to $0.5069 \mathrm{D}$ for $\mathrm{Kf}$ and from -0.3705 to $0.5638 \mathrm{D}$ for Ks). Pan et al. [32] assessed the agreement of ray-traced TCP using a Pentacam, Sirius Scheimpflug-Placido topographer (CSO, Firenze, Italy), and Galilei dual Scheimpflug analyzer (Ziemer, Port, Switzerland) in 74 eyes of 74 healthy subjects. They reported that the single Scheimpflug camera, Pentacam, and Sirius, showed an almost identical result in TCP with a $95 \%$ LoA of -0.45 to $0.51 \mathrm{D}$, which is clinically acceptable. Nonetheless, the dual Scheimpflug camera showed a higher value, and they said the difference in principle (single or dual Scheimpflug) of the devices contributed to this difference in results. Savini et al. [33] compared anterior segment values using the AS-OCT device MS-39 (CSO, Florence, Italy) and Pentacam HR and Sirius. The results showed that MS-39 cannot be considered interchangeable due to moderate agreement. Sel et al. [34] compared Pentacam AXL and IOL Master 700 in 50 eyes, and concluded that J0 and J45 values are interchangeable with mean difference of $0.02 \pm 0.11$ for J0 ( $p=0.115), 0.02 \pm 0.10(p=0.255)$ with narrow $95 \%$ LoA; -0.18 to 0.23 for J0, -0.18 to 0.21 for J45. However, $\mathrm{Km}$, anterior chamber depth, and AXL are not interchangeable between two devices. In line with previous studies, the keratometric values were not interchangeable, 
showing a wide range of $95 \% \mathrm{LoA}$ in anterior $\mathrm{K}(0.68$ to $0.80 \mathrm{D})$, posterior K ( 0.25 to $0.31 \mathrm{D})$, and TCP (0.79 to 1.10 D) in this study. However, vectoral components were interchangeable, showing a narrow range of $95 \%$ LoA in anterior J0 ( -0.27 to 0.13$)$, anterior $\mathrm{J} 45$ ( -0.29 to 0.10$)$, posterior $\mathrm{J} 0$ (-0.07 to 0.08$)$, posterior J45 (-0.07 to 0.12$)$, TCP J0 (-0.32 to 0.11 ), and TCP J45 (-0.28 to 0.09$)$.

Regarding corneal HOAs, previous studies have reported the results of comparing HOAs measured by different devices. Piccinini et al. [35] compared the HOAs using the Pentacam HR and Galilei G4, which were based on a dual Scheimpflug camera with a placido system in 105 eyes of 105 subjects. Total RMS, coma, and trefoil showed significant difference $(p<0.001)$ but not in spherical aberration ( $p=0.125)$. The mean differences (95\% LoA range) were $-0.0034 \pm 0.101 \mu \mathrm{m}(-0.233$ to 0.166$)$ for the oblique trefoil, $0.052 \pm 0.103 \mu \mathrm{m}$ ( -0.150 to 0.254$)$ for the horizontal trefoil, $0.152 \pm 0.141 \mu \mathrm{m}(-0.125$ to 0.427$)$ for the horizontal coma, $-0.055 \pm 0.139 \mu \mathrm{m}(-0.328$ to 0.217$)$ for the vertical coma, and $0.012 \pm 0.081 \mu \mathrm{m}(-0.159$ to 0.159$)$ for the spherical aberration, and $1.238 \pm 0.677 \mu \mathrm{m}(-0.536$ to 1.081$)$ for the total RMS, showing reasonable correlations and narrow $95 \%$ LoA between two devices, all of which suggest these measurements can be considered equivalent for a clinical setting. Kim et al. [36] compared the measurement of 45 eyes using a color LED topographer Cassini, and Pentacam HR. Among the measurements, spherical aberration, coma, trefoil, tetrafoil, and astigmatism of anterior, posterior and total keratometry did not differ between the two devices, but there were significant differences in the mean of anterior and total keratometry and the axis of astigmatism of anterior cornea curvature. In the agreement analysis, total corneal astigmatism and vector component J0, J45 showed a low degree of agreement (95\% LoA: -0.845 to $1.125 \mathrm{D}$ for astigmatism, -2.415 to $2.235 \mathrm{D}$ for J0, -1.389 to $0.989 \mathrm{D}$ for J45). Shin et al. [37] compared corneal curvature and HOAs using a Placid-based videokeratoscope Keratron scout (Optikon, Rome, Italy) and Pentacam HR in 46 eyes of 23 patients. They reported that there was no statistically significant difference for the anterior $\mathrm{Ks}, \mathrm{Kf}$, and $\mathrm{Km}$ and total RMS, corneal spherical aberration, but not in the coma of a total cornea ( $p=0.005)$, and the trefoil of an anterior and total cornea $(p<0.001)$. In the present study, vertical coma, horizontal trefoil, spherical aberration, and each RMS of the fifth and sixth order showed a statistically significant difference. Nevertheless, there were moder- ate or higher correlations and an acceptable 95\% LoA range in all HOAs except the horizontal trefoil and RMS of the fifth order and RMS of sixth order. The values of HOAs decreased as the order of the Zernike coefficient increased as others have shown [38]. Especially in ANTEIRION, the RMS of the fifth and sixth order HOAs showed up as zero in many cases. This may affect the wide range of the $95 \%$ LoA in RMS of the fifth and sixth order HOAs in this study.

In this study, the two devices were interchangeable in some, but not interchangeable in others. Since both devices are expensive, it is not common to have both devices in one clinic. And ophthalmologists are often faced with the task of interpreting results that have been tested in other clinics against the results of their own instruments. Thus, it is necessary to compare and investigate the interchangeability of results of two different devices in a clinical setting. Considering the cause of the difference in corneal measurements between two devices, the most likely cause is the difference in measurement principles. Above all, there is a difference in light source between Scheimpflug camera, which obtains an image of anterior segment by emitting a blue LED with a wavelength of $450 \mathrm{~nm}$, and OCT-based technology using light with a wavelength of $1,300 \mathrm{~nm}$. ANTERION take a shorter time for image acquisition than Pentacam HR, which may be less chance of giving irritation to the subjects. In addition, ANTERION applies an eye-tracking technology during all measurements. These differences may affect the differences in results of the two devices in the present study.

This study has a few limitations. First, the number of subjects was relatively small, and adults aged only 20 to 60 were included. Second, since the measurement was not repeatedly performed for each device, the measurement error for the value itself cannot be excluded. However, both devices have shown good repeatability in other previous studies $[16,33,39]$. Also, image quality is always checked during image acquisition. In addition, all the participants in this study were well cooperated with no underlying medical/ophthalmological history and fully understood the examination method, so examinations proceeded easily with an experienced examiner. Thus, it is thought that the measurement error would have been small. Third, comparisons were not made according to the degree of refractive error, which can affect HOAs [38]. Fourth, patients who had corneal diseases such as keratoconus or underwent 
corneal refractive surgery were not evaluated. Further studies with patients of various age groups, refractive errors, and corneal conditions would be necessary. In addition, as there are more devices based on various principles, it is necessary to use them to compare anterior segment measurements together.

In conclusion, our study is the first to compare the keratometry, including TCP and HOAs, between ANTERION and Pentacam HR. Our results showed that there are statistically significant differences in keratometic measurement not acceptable for interchangeable use. However, HOAs showed acceptable agreement, except for horizontal trefoil and RMS of the fifth and sixth order.

\section{Conflict of Interest}

No potential conflict of interest relevant to this article was reported.

\section{References}

1. Konstantopoulos A, Hossain P, Anderson DF. Recent advances in ophthalmic anterior segment imaging: a new era for ophthalmic diagnosis? Br J Ophthalmol 2007;91:551-7.

2. Chen W, McAlinden C, Pesudovs K, et al. Scheimpflug-Placido topographer and optical low-coherence reflectometry biometer: repeatability and agreement. $J$ Cataract Refract Surg 2012;38:1626-32.

3. Ventura BV, Wang L, Ali SF, et al. Comparison of corneal power, astigmatism, and wavefront aberration measurements obtained by a point-source color light-emitting diode-based topographer, a Placido-disk topographer, and a combined Placido and dual Scheimpflug device. J Cataract Refract Surg 2015;41:1658-71.

4. Fabian E, Wehner W. Prediction accuracy of total keratometry compared to standard keratometry using different intraocular lens power formulas. J Refract Surg 2019;35:362-8.

5. Lawless M, Jiang JY, Hodge C, et al. Total keratometry in intraocular lens power calculations in eyes with previous laser refractive surgery. Clin Exp Ophthalmol 2020;48:749-56.

6. Reitblat O, Levy A, Kleinmann G, et al. Effect of posterior corneal astigmatism on power calculation and alignment of toric intraocular lenses: comparison of methodologies. $J$ Cataract Refract Surg 2016;42:217-25.
7. Savini G, Naeser K. An analysis of the factors influencing the residual refractive astigmatism after cataract surgery with toric intraocular lenses. Invest Ophthalmol Vis Sci 2015;56:827-35.

8. Savini G, Naeser K, Schiano-Lomoriello D, Ducoli P. Optimized keratometry and total corneal astigmatism for toric intraocular lens calculation. J Cataract Refract Surg 2017;43:1140-8.

9. Kern C, El Kaissi L, Kortuem K, et al. Comparing refractive outcomes of a standard industry toric IOL calculator using anterior corneal astigmatism and total corneal refractive power. Graefes Arch Clin Exp Ophthalmol 2020; 258:345-50.

10. Gordon-Shaag A, Millodot M, Ifrah R, Shneor E. Aberrations and topography in normal, keratoconus-suspect, and keratoconic eyes. Optom Vis Sci 2012;89:411-8.

11. Pinero DP, Nieto JC, Lopez-Miguel A. Characterization of corneal structure in keratoconus. J Cataract Refract Surg 2012;38:2167-83.

12. Yamaguchi T, Dogru M, Yamaguchi K, et al. Effect of spherical aberration on visual function under photopic and mesopic conditions after cataract surgery. $J$ Cataract Refract Surg 2009;35:57-63.

13. Iseli HP, Jankov M, Bueeler M, et al. Corneal and total wavefront aberrations in phakic and pseudophakic eyes after implantation of monofocal foldable intraocular lenses. $J$ Cataract Refract Surg 2006;32:762-71.

14. Song IS, Kim MJ, Yoon SY, et al. Higher-order aberrations associated with better near visual acuity in eyes with aspheric monofocal IOLs. J Refract Surg 2014;30:442-6.

15. Moshirfar M, Thomson AC, Thomson RJ, et al. Use of presbyopia-correcting intraocular lenses in patients with prior corneal refractive surgery. Curr Opin Ophthalmol 2021;32:45-53.

16. Tana-Rivero P, Aguilar-Corcoles S, Ruiz-Mesa R, Montes-Mico R. Repeatability of whole-cornea measurements using a new swept-source optical coherence tomographer. Eur J Ophthalmol 2021;31:1709-19.

17. Montes-Mico R, Tana-Rivero P, Aguilar-Corcoles S, Ruiz-Mesa R. Assessment of anterior segment measurements using a high-resolution imaging device. Expert Rev Med Devices 2020;17:969-79.

18. Ruiz-Mesa R, Aguilar-Corcoles S, Montes-Mico R, Tana-Rivero P. Ocular biometric repeatability using a new high-resolution swept-source optical coherence tomographer. Expert Rev Med Devices 2020;17:591-7. 
19. Fisus AD, Hirnschall ND, Findl O. Comparison of 2 sweptsource optical coherence tomography-based biometry devices. J Cataract Refract Surg 2021;47:87-92.

20. Pardeshi AA, Song AE, Lazkani N, et al. Intradevice repeatability and interdevice agreement of ocular biometric measurements: a comparison of two swept-source anterior segment OCT devices. Transl Vis Sci Technol 2020;9:14.

21. Tana-Rivero P, Aguilar-Corcoles S, Rodriguez-Prats JL, et al. Agreement of white-to-white measurements with sweptsource OCT, Scheimpflug and color LED devices. Int Ophthalmol 2021;41:57-65.

22. Tana-Rivero P, Aguilar-Corcoles S, Tello-Elordi C, et al. Agreement between 2 swept-source OCT biometers and a Scheimpflug partial coherence interferometer. $J$ Cataract Refract Surg 2021;47:488-95.

23. Fan R, Chan TC, Prakash G, Jhanji V. Applications of corneal topography and tomography: a review. Clin Exp Ophthalmol 2018;46:133-46.

24. McAlinden C, Schwiegerling J, Khadka J, Pesudovs K. Corneal aberrations measured with a high-resolution Scheimpflug tomographer: repeatability and reproducibility. J Cataract Refract Surg 2020;46:581-90.

25. Asam JS, Polzer M, Tafreshi A, et al. Anterior segment OCT. In: Bille JF, editor. High resolution imaging in microscopy and ophthalmology: new frontiers in biomedical optics. Cham: Springer; 2019. p. 285-99.

26. Thibos LN, Wheeler W, Horner D. Power vectors: an application of Fourier analysis to the description and statistical analysis of refractive error. Optom Vis Sci 1997;74:367-75.

27. Thibos LN, Horner D. Power vector analysis of the optical outcome of refractive surgery. $J$ Cataract Refract Surg 2001;27:80-5.

28. Klijn S, Reus NJ, van der Sommen CM, Sicam VA. Accuracy of total corneal astigmatism measurements with a Scheimpflug imager and a color light-emitting diode corneal topographer. Am J Ophthalmol 2016;167:72-8.

29. Park DY, Lim DH, Hwang S, et al. Comparison of astigmatism prediction error taken with the Pentacam measurements, Baylor nomogram, and Barrett formula for toric in- traocular lens implantation. BMC Ophthalmol 2017;17:156.

30. Ozyol P, Ozyol E. Agreement between swept-source optical biometry and Scheimpflug-based topography measurements of anterior segment parameters. Am J Ophthalmol 2016;169:73-8.

31. Cui XH, Yoo YS, An Y, Joo CK. Comparison of keratometric measurements between color light-emitting diode topography and Scheimpflug camera. BMC Ophthalmol 2019;19:98

32. Pan C, Tan W, Savini G, et al. A comparative study of total corneal power using a ray tracing method obtained from 3 different Scheimpflug camera devices. Am J Ophthalmol 2020;216:90-8.

33. Savini G, Schiano-Lomoriello D, Hoffer KJ. Repeatability of automatic measurements by a new anterior segment optical coherence tomographer combined with Placido topography and agreement with 2 Scheimpflug cameras. J Cataract Refract Surg 2018;44:471-8.

34. Sel S, Stange J, Kaiser D, Kiraly L. Repeatability and agreement of Scheimpflug-based and swept-source optical biometry measurements. Cont Lens Anterior Eye 2017;40:318-22.

35. Piccinini AL, Golan O, Hafezi F, Randleman JB. Higher-order aberration measurements: comparison between Scheimpflug and dual Scheimpflug-Placido technology in normal eyes. J Cataract Refract Surg 2019;45:490-4.

36. Kim DY, Ha M, Yi R, et al. Comparison of corneal astigmatism and higher-order aberrations between color light-emitting diode topographer and Scheimpflug imager. $J$ Korean Ophthalmol Soc 2019;60:922-8.

37. Shin JY, Lee MY, Chung SH. Comparison of keratometry and corneal higher order aberrations between Scout videokeratoscope and Pentacam Scheimpflug Camera. J Korean Ophthalmol Soc 2014;55:1758-64.

38. Karimian F, Feizi S, Doozande A. Higher-order aberrations in myopic eyes. J Ophthalmic Vis Res 2010;5:3-9.

39. McAlinden C, Khadka J, Pesudovs K. A comprehensive evaluation of the precision (repeatability and reproducibility) of the Oculus Pentacam HR. Invest Ophthalmol Vis Sci 2011;52:7731-7. 